\title{
Genetic Discovery for Congenital Heart Defects
}

\author{
Bruce D. Gelb
}

\begin{abstract}
Congenital heart disease (CHD) behaves like a complex genetic trait in most instances. Recent advances in genomics have provided tools for uncovering genetic variants underlying complex traits that are now being applied to study CHD. Massively parallel DNA sequencing has shown that de novo mutations contribute to $\sim 10 \%$ of severe $\mathrm{CHD}$ and implicated chromatin remodeling in pathogenesis. Genome scanning methods for copy number variants (CNVs) identify likely pathogenic genomic alterations in $10 \%$ of infants with hypoplastic left heart syndrome and related single ventricle forms of CHD. The growth and neurocognitive development of children with CHD and those CNVs is worse, and clinical examination is relatively insensitive for detecting those CNVs. In sum, new opportunities for preventing and ameliorating CHD and its comorbidities are anticipated as its genetic architecture is elaborated through the use of state-of-the-art genomic approaches.
\end{abstract}

\section{Keywords}

Congenital heart disease $\bullet$ Copy number variants $\bullet$ Exome sequencing $\bullet$ Genetics

\subsection{Introduction}

Congenital heart defects (CHD), with an estimated incidence of 2-3\% when bicuspid aortic valve (BAV) is included, are widely believed to have strong genetic underpinnings. Epidemiologic studies have shown considerable consistency in the

\footnotetext{
B.D. Gelb, M.D. ( $\bowtie)$

Mindich Child Health and Development Institute, Departments of Pediatrics and Genetics and Genomics Sciences, Icahn School of Medicine at Mount Sinai, One Gustave Levy Place, Box 1040, New York, NY 10029, USA

e-mail: bruce.gelb@mssm.edu
}

T. Nakanishi et al. (eds.), Etiology and Morphogenesis of Congenital Heart Disease, DOI 10.1007/978-4-431-54628-3_51 
distribution of CHD lesions across time and geographic location. The landmark study by Ruth Whittemore, in which she examined recurrence risks for offspring of women with CHD, revealed a $16 \%$ rate with a $60 \%$ concordance in the form of CHD between mother and child [1]. Estimates of heritability for BAV and hypoplastic left heart syndrome (HLHS) are $89 \%$ and $95 \%$, respectively [2, 3]. Recent studies of CHD from Denmark, where the highly organized medical system enables population studies, have provide estimates of the relative risks of various forms of CHD among first-degree relatives that significantly increased, often to $>5$ [4]. Taken as a whole, these epidemiologic findings point to genetic defects contributing importantly to CHD etiology.

Identification of the precise mutations has been challenging [5]. We have known for some time that a modest percentage of $\mathrm{CHD}(\sim 5 \%)$ is attributable to aneuploidies such as trisomy 21 . With the advent of molecular genetic approaches, point mutations with apparently strong effects have been identified in rare families inheriting CHD in Mendelian or near-Mendelian fashion. More recently, the role of larger genomic events generating pathologic copy number variants (CNVs) for CHD has become apparent. This started with the recognition of 22q11 deletions underlying DiGeorge, Takao conotruncal face, and velocardiofacial syndromes. Several surveys have implicated a wide range of gain and loss CNVs in various forms of CHD.

Based on the author's oral presentation at the 2013 Takao Symposium, two recent studies that further elaborate the genetic etiology of CHD will be reviewed here.

\subsection{De Novo Mutations}

Through recent advances in molecular genetic technologies, it is now possible to sequence the roughly $1 \%$ of the human genome that contains the coding regions for all genes (called the exome), representing approximately 180,000 exons and 30 megabases $(\mathrm{Mb})$, in a relatively rapid and affordable manner. While exome sequencing was initially used to discover mutations underlying Mendelian disorders, current efforts are increasingly focusing on unraveling complex genetic traits.

The Pediatric Cardiac Genomics Consortium (PCGC) [6], a National Heart, Lung, and Blood Institute-funded research enterprise, recently completed a firstof-kind study to determine the role of de novo mutations in the etiology of severe forms of CHD [7]. Exome sequencing was performed for 362 parent-offspring trios, in which the offspring had a sporadic conotruncal defect, left ventricular outflow track obstructive lesion, or heterotaxy, and compared to comparable data from 264 control trios. While the overall rate of de novo point and small insertion/ deletion (indel) changes was equivalent between CHD cases and controls, there was an excess burden of protein-altering mutations in genes highly expressed during heart development (odds ratio (OR) of 2.53). Excess mutations had a role in $10 \%$ of CHD cases and led to the estimate that 400 genes underlie these birth defects. After filtering to retain variants most likely to be deleterious (nonsense, 
splice site, and frameshift defects), the burden among CHD cases increased, attaining an OR of 7.50.

Next, the PCGC investigators asked whether the burden of de novo proteinaltering mutations among the CHD cases preferentially targeted particular biologic processes [7]. Indeed, they observed a highly significant enrichment of mutation among genes encoding proteins relevant for chromatin biology, specifically the production, removal, or reading of methylation of Lys4 of histone 3 (H3K4me) (Fig. 51.1). The phenotypes of the eight subjects harboring H3K4me de novo mutations were diverse, both with respect to the form of CHD and the involvement of extracardiac tissues. In addition, two independent de novo mutations were found in SMAD2, which encodes a protein with relevance for demethylation of Lys27 of histone 3 (H3K27me). SMAD2 contributes to the development of the left-right body axis; both subjects harboring SMAD2 mutations had dextrocardia with unbalanced complete atrioventricular canal defects with pulmonic stenosis. While the contribution of chromatin remodeling to cardiovascular development generally and

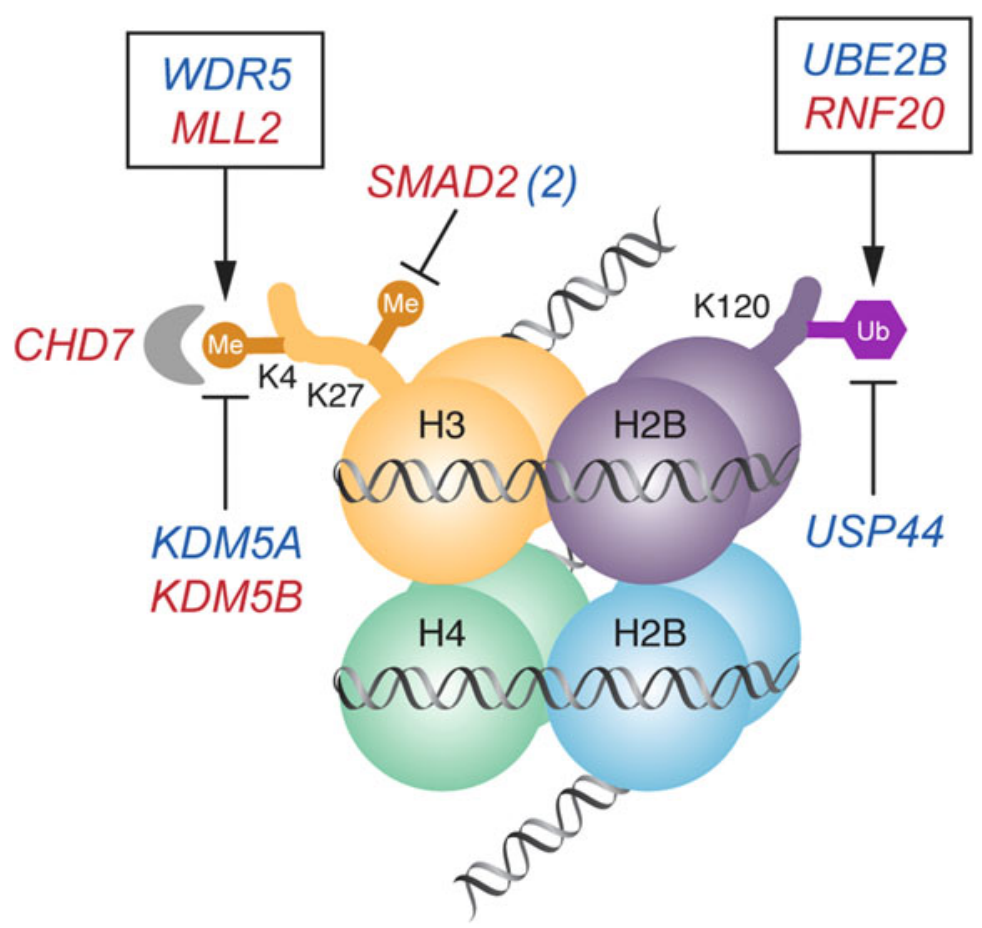

Fig. 51.1 De novo mutations in the H3K4 and H3K27 methylation pathways. Nucleosome with histone octamer and DNA, H3K4 methylation bound by CHD7, and H3K27 methylation and H2BK120 ubiquitination is shown. Genes mutated in CHD that affect the production, removal, and reading of these histone modifications are shown; genes with damaging mutations are shown in red, and those with missense mutations are shown in blue. SMAD2 (2) indicates there are two patients with a mutation in this gene. Genes whose products are found together in a complex are enclosed in a box (Reprinted without modification from Ref. [7]) 
certain rare genetic syndromes with CHD like Kabuki syndrome had been recognized previously, this study exposed a far broader role in CHD pathogenesis. The finding also suggests a fascinating potential link to other birth defects as de novo chromatin remodeling mutations have also been implicated in autism [8].

\subsection{Copy Number Variants}

CNVs, which are gains or losses of DNA ranging in size from $1 \mathrm{~kb}$ to several Mbs, affect roughly $10 \%$ of the human genome [9]. CNVs are typically detected on a genome-wide basis using SNP microarrays or array comparative genomic hybridization $(\mathrm{aCGH})$. Although differentiating pathogenic CNVs from benign polymorphic one remains challenging, it has become clear that pathologic CNVs contribute significantly to the pathogenesis of $\mathrm{CHD}$ as rare large $\mathrm{CNVs}$ are observed in 5-15\% of affected individuals [10-14].

To address the issue of how pathogenic CNVs affected outcomes for children with CHD, Carey and colleagues studied children who had previously been subjects in one or both of two clinical trials undertaken by the National Heart, Lung, and Blood Institute-funded Pediatric Heart Network [15]. The two studies were the Infants with Single Ventricle (ISV) and Single Ventricle Reconstruction (SVR) trials, both focusing primarily on infants with HLHS. Carey et al. used aCGH to identify CNVs in 223 subjects from the ISV and SVR trials for whom genomic DNA was available. Because the DNA samples were procured late in those trials, subjects who died earlier, particularly perioperatively, could not be studied. For the CNV work, CNVs were $\geq 300 \mathrm{~kb}$ and not identified as genetic polymorphisms based on existing databases.

Carey and co-workers observed pathogenic CNVs in $13.9 \%$ of the children, which was significantly more than the $4.4 \%$ rate in the controls (preexisting data from blood samples from subjects with solid cancers from The Cancer Genome Atlas project) [15]. For the CHD cases, there was a roughly 4:1 ratio of duplications to deletions and the median sizes were $674 \mathrm{~kb}$ and $1.5 \mathrm{Mb}$, respectively. Parental genomic DNAs were available for 12 of the subjects with pathogenic CNVs; analysis of those showed that slightly more than have of the pathogenic CNVs were inherited.

For the ISV and SVR studies, careful assessment of growth (weight-, height- and head circumference-for-age z-scores) and neurocognitive function (Mental Developmental Index (MDI) and Psychomotor Developmental Index (PDI) measured with the Bayley Scales of Infant Development II) were determined at 14 months of age [15]. Comparison of the CHD subjects harboring pathogenic CNVs to those who did not revealed that the former were significantly shorter by an average of $0.65 \mathrm{z}$-score. Subgroup analysis showed that those with deletion CNVs had significantly lower PDI scores.

Among the 31 pathogenic CNVs found among the CHD subjects, 13 had previously been associated with genomic disorders. The children harboring these known CNVs had the worst outcomes with globally reduced neurocognitive development (MDI and PDI) as well as the slowest growth (Table 51.1). 
Table 51.1 Fourteen-month outcomes for subgroups based on genetic examination

\begin{tabular}{|c|c|c|c|c|c|c|}
\hline & $N^{\mathrm{a}}$ & MDI & PDI & Weight $Z$ & Length $Z$ & $\mathrm{HC} Z$ \\
\hline \multirow{2}{*}{$\begin{array}{l}\text { CNV- Syndrome- Dysmorphic- } \\
\text { Extracardiac- }\end{array}$} & \multirow[t]{2}{*}{69} & 89.1 & 77.5 & -0.71 & -1.13 & -0.34 \\
\hline & & $(18.0)$ & $(20.2)$ & $(1.07)$ & $(1.32)$ & $(1.24)$ \\
\hline \multirow[t]{2}{*}{$\mathrm{CNV}+$} & \multirow[t]{2}{*}{14} & 85.4 & $65.1^{*}$ & -0.94 & -1.61 & -0.04 \\
\hline & & $(20.1)$ & $(17.6)$ & $(0.88)$ & $(1.08)$ & $(1.38)$ \\
\hline \multirow[t]{2}{*}{$\mathrm{CNV}+$ or syndrome } & \multirow[t]{2}{*}{18} & 83.2 & $67.9^{*}$ & $-1.19^{*}$ & $-1.99^{*}$ & -0.16 \\
\hline & & (18.7) & $(19.4)$ & $(1.11)$ & (1.73) & $(1.27)$ \\
\hline \multirow[t]{2}{*}{ Dysmorphic/extracardiac } & \multirow[t]{2}{*}{29} & 89.3 & 78.1 & -0.63 & -1.18 & -0.10 \\
\hline & & $(18.1)$ & $(20.0)$ & $(1.25)$ & $(1.30)$ & $(1.28)$ \\
\hline \multirow[t]{2}{*}{ CNV syndrome } & \multirow[t]{2}{*}{98} & 89.4 & 77.8 & -0.73 & -1.18 & -0.25 \\
\hline & & $(17.5)$ & $(19.8)$ & $(1.10)$ & $(1.43)$ & $(1.32)$ \\
\hline
\end{tabular}

All data shown as mean (standard deviation)

$M D I$ mental developmental index, PDI psychomotor developmental index, Weight $Z$ weight-forage $\mathrm{Z}$ score at 14 months, Length $Z$ length-for-age $Z$ score at 14 months, $H C Z$ head circumference $\mathrm{Z}$ score at 14 months

${ }^{*} p<0.05$ compared to $\mathrm{CNV} /$ syndrome/dysmorphic/extracardiac

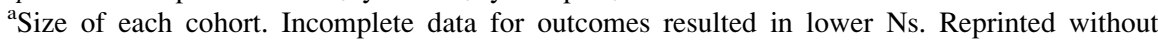
modification from Ref. [15]

Finally, Carey and co-workers looked at the sensitivity of clinical examination in detecting children with CHD and pathogenic CNVs, which was possible for the subjects from the SVR study [15]. Of 116 children examined, $3.4 \%$ were diagnosed with a defined genetic syndrome, none associated with a pathogenic $\mathrm{CNV}$, and $25 \%$ had one or more dysmorphic features and/or extracardiac malformations, which were not enriched among those with CNVs. Most strikingly, more than $70 \%$ of the children with CNVs previously associated with genomic disorders had no dysmorphic feature or extracardiac anomaly. Taken as a whole, this analysis showed that clinical examination was relatively insensitive for determining which children with CHD harbored pathogenic CNVs.

The findings from this study support the routine use of CNV testing in newborns with single ventricle forms of CHD to enable better prognostication and early intervention. Similarly, the poorer linear growth associated with all pathogenic CNVs, the worse neurocognitive outcomes with deletions, and particularly the globally poor outcomes with CNVs associated with known genomic disorders could impact clinical trial outcomes depending on the designated endpoints.

\subsection{Future Directions}

Recent advances in genomics have enabled the elucidation of the architecture of CHD genetics. As that project proceeds, the next challenge will be translating those gene discoveries into actionable clinical approaches. Improved prognostication with respect to the heart disease and extracardiac comorbidities can be used most immediately. Finding strategies that reduce CHD incidence or alter its natural 
history will require the elucidation of pathogeneses, and a careful balancing of potential benefits with adverse effects as fundamental biological process like chromatin remodeling will probably predominate.

Acknowledgments The author declares that he has no conflict relevant to this manuscript. Funding for this work was partly from a grant from NIH (U01 HL071207) to B.D.G.

Open Access This chapter is distributed under the terms of the Creative Commons AttributionNoncommercial 2.5 License (http://creativecommons.org/licenses/by-nc/2.5/) which permits any noncommercial use, distribution, and reproduction in any medium, provided the original author(s) and source are credited.

The images or other third party material in this chapter are included in the work's Creative Commons license, unless indicated otherwise in the credit line; if such material is not included in the work's Creative Commons license and the respective action is not permitted by statutory regulation, users will need to obtain permission from the license holder to duplicate, adapt or reproduce the material.

\section{References}

1. Whittemore R, Hobbins JC, Engle MA. Pregnancy and its outcome in women with and without surgical treatment of congenital heart disease. Am J Cardiol. 1982;50:641-51.

2. Cripe L, Andelfinger G, Martin LJ, et al. Bicuspid aortic valve is heritable. J Am Coll Cardiol. 2004;44:138-43.

3. Hinton Jr RB, Martin LJ, Tabangin ME, et al. Hypoplastic left heart syndrome is heritable. J Am Coll Cardiol. 2007;50:1590-5.

4. Oyen N, Poulsen G, Boyd HA, et al. Recurrence of congenital heart defects in families. Circulation. 2009;120:295-301.

5. Fahed AC, Gelb BD, Seidman JG, Seidman CE. Genetics of congenital heart disease: the glass half empty. Circ Res. 2013;112:707-20.

6. Gelb B, Brueckner M, Chung W, et al. The Congenital Heart Disease Genetic Network Study: rationale, design, and early results. Circ Res. 2013;112:698-706.

7. Zaidi S, Choi M, Wakimoto $\mathrm{H}$, et al. De novo mutations in histone-modifying genes in congenital heart disease. Nature. 2013;498:220-3.

8. O'Roak BJ, Vives L, Fu W, et al. Multiplex targeted sequencing identifies recurrently mutated genes in autism spectrum disorders. Science. 2012;338:1619-22.

9. Redon R, Ishikawa S, Fitch KR, et al. Global variation in copy number in the human genome. Nature. 2006;444:444-54.

10. Thienpont B, Mertens L, de Ravel T, et al. Submicroscopic chromosomal imbalances detected by array-CGH are a frequent cause of congenital heart defects in selected patients. Eur Heart J. 2007;228:2778-84.

11. Greenway SC, Pereira AC, Lin JC, et al. De novo copy number variants identify new genes and loci in isolated sporadic tetralogy of Fallot. Nat Genet. 2009;41:931-5.

12. Silversides CK, Lionel AC, Costain G, et al. Rare copy number variations in adults with tetralogy of Fallot implicate novel risk gene pathways. PLoS Genet. 2012;8:e1002843.

13. Hitz MP, Lemieux-Perreault LP, Marshall C, et al. Rare copy number variants contribute to congenital left-sided heart disease. PLoS Genet. 2012;8:e1002903.

14. Fakhro KA, Choi M, Ware SM, et al. Rare copy number variations in congenital heart disease patients identify unique genes in left-right patterning. Proc Natl Acad Sci U S A. 2011;108:2915-20.

15. Carey AS, Liang L, Edwards J, et al. Effect of copy number variants on outcomes for infants with single ventricle heart defects. Circ Cardiovasc Genet. 2013;6:444-51. 